\title{
Tapetoretinal Degeneration in Childhood Presenting as a Disturbance of Behaviour
}

\author{
BRIAN HARCOURT, DAVID HOPKINS
}

British Medical fournal, 1972, 1, 202-205

\section{Summary}

Four children presented at the age of 6 years with progressive disturbances of behaviour due to increasing visual handicap. In three of them routine ophthalmological examination was carried out in the early stages, but no objective evidence of eye disease was found. The severity and nature of the visual defect and its causal relationship to the behaviour disorder went unrecognized for periods ranging from 18 to 30 months. Eventually the development of retinal changes enabled a diagnosis of tapetoretinal degeneration to be made which was confirmed by the finding of extinction of the electroretinographic response. Transfer of the children to schools for the visually handicapped resulted in improvement in their behaviour disorder.

Electroretinography is of great importance in the early diagnosis of juvenile tapetoretinal degeneration.

\section{Introduction}

The term "tapetoretinal degeneration" was first used by Leber (1916) to describe a group of hereditary degenerative disorders affecting the retina and in some cases the choroid of the eyes; "tapeto-" here refers to the tapetum nigrum (pigment epithelium of the retina) and not to the choroidal tapetum lucidum, a light-reflecting layer present principally in nocturnal animals. This descriptive term was introduced because it was thought that the pathological changes affected mainly this retinal layer, but it is now known that they are in fact much more widespread, with progressive degeneration of all the retinal layers, often associated with pigment proliferation and choroidal atrophy. A number of different forms can be recognized clinically and pathologically, the best known being the primary pigmentary retinal dystrophies typified by retinitis pigmentosa. The ocular degeneration is most commonly an isolated phenomenon, but it may also occur as one important aspect of a number of interesting syndromes characterized usually by major neurological features (Hallgren's syndrome, Laurence-Moon-Biedl syndrome); tapetoretinal degeneration is also a late manifestation of certain hereditary lipid disorders, in particular of Refsum's disease and abetalipoproteinaemia (Bassen-Kornzweig syndrome).

Congenital, juvenile, and adult forms of tapetoretinal degeneration are recognized. Retinitis pigmentosa is the classic adult form and is usually first manifest by symptoms of night blindness in the second decade of life. As the subsequent slowly progressive visual symptoms are accompanied by strikingly abnormal ophthalmoscopic appearances it is usually possible to make the diagnosis by clinical examination alone. In contrast, in the congenital form of the disorder the ophthalmoscopic appearances, in the early years of life at least, are not strikingly abnormal, but a very severe defect

General Infirmary, Leeds

BRIAN HARCOURT, F.R.C.s., D.o., Consultant Ophthalmic Surgeon

Royal Infirmary, Bradford

DAVID HOPKINS, F.R.C.s., D.o., Consultant Ophthalmic Surgeon in both central and peripheral vision is present from birth or early infancy, giving rise to a pendular nystagmus or roving eye movements and to a pronounced impairment of the pupillary light reflexes.

Juvenile cases are characterized by normal visual function in the first few years of life followed by a slowly progressive deterioration, with the onset of visual symptoms commonly between the ages of 5 and 8 years. Macular function is affected early, with impairment of central vision, but, as is the rule in cases of visual defect from whatever cause acquired after the age of 5, pendular nystagmus is not a feature. As in the congenital form, there are few or no signs of retinal abnormality on ophthalmoscopic examination in the early stages, apart from some retinal arterial attenuation, and an affected child may therefore suffer from a severe visual handicap which, in the absence of any obvious ocular cause, may be underestimated. Fortunately the diagnosis can be made with complete certainty by electroretinography, as an early and pronounced reduction or extinction of the electroretinogram (E.R.G.) response is a constant feature of all forms of widespread tapetoretinal degeneration (Burian, 1962; Goodman et al., 1963; Harcourt, 1970).

Electroretinography is a technique for assessing the mass electrical response of the retina to a bright light stimulus. An active electrode carried in a corneal contact lens and indifferent electrodes on the skin of the face (Fig. 1) are placed

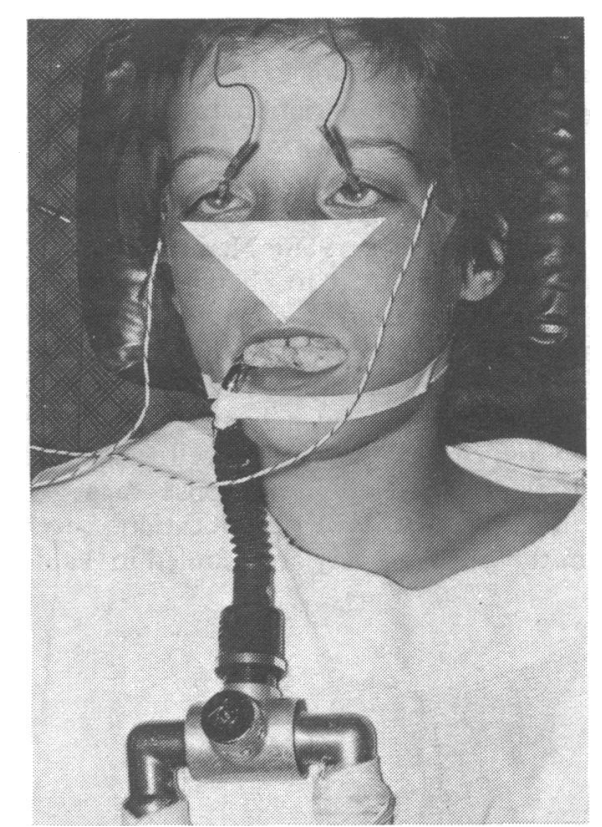

FIG. 1-Electroretinography under general anaesthesia showing electrode positions.

in circuit with an amplifier and recorder. In adults and older children the investigation is carried out with topical corneal anaesthesia, but in early childhood, and particularly with disturbed patients, a general anaesthetic is advisable. The pupils are fully dilated for the investigation, which can then be combined with a full examination of all the ocular structures. 
The normal E.R.G. (Fig. 2) has two principal features (Karpe, 1945): an a-wave of negative potential, which is thought to represent activity in the outer segments of the retinal receptors (rods and cones), and a b-wave of positive potential which is due principally to electrical changes occurring in the bipolar cells of the retina (Noell, 1959). As the E.R.G. is a mass response representing the summation of electrical activity in the whole of the outer layers of the retina where the rods are predominant, the response increases in amplitude during dark adaptation, when the rods become more active. After full dark adaptation the amplitude of the E.R.G. response is of the order of 250 $\mu \mathrm{V}$ in normal subjects, but in patients with tapetoretinal degeneration the response is grossly diminished or absent.

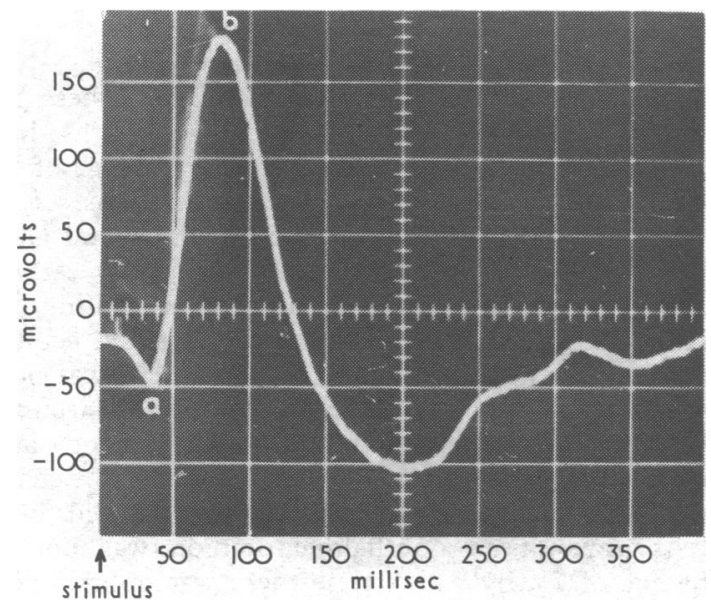

FIG. 2-Electroretinogram of a dark-adapted healthy 5-year-old child.

In juvenile cases of tapetoretinal degeneration the insidious loss of vision, commonly first manifest when the child starts school, may lead to bizarre disturbances of behaviour. As the eyes appear grossly normal on routine clinical examination the nature of the ocular disorder may be temporarily overlooked, and the severity of the visual defect may be masked by the lack of co-operation of the young patient when attempts are made to test the visual acuity. It is the purpose of this communication to present the histories of four such young children whom we have examined during the past three years and to emphasize the critical importance of electroretinography in the early and exact diagnosis of the disorder. In each case the child was born at term after a normal pregnancy, the parents were unrelated, and there was no relevant family or social history.

\section{Case 1}

This girl's development was unremarkable until the age of 6 years, when her school progress became very slow and poorly maintained and she became increasingly disinclined to enter into relationships with other children and adults. She took little interest in her surroundings, cried for a lot of the time, and started smearing lavatory walls with faeces. At home she imposed great strains on the family, continuously talking and showing an intense jealousy towards her infant sister, whom she would attack if no one was around. The mother had noticed that the child had always been much more afraid of the dark than her siblings and that she had recently started to cry a lot at night and sleep very little. The parents attributed these disturbances to attention seeking.

Referral to a psychiatrist at the age of 7 revealed a gross discrepancy between her verbal scale intelligence quotient (I.Q.) of 72 and her performance scale I.Q. of 99, providing further evidence of her reluctance to communicate. The full scale I.Q. was 83. It was considered that she was semi-autistic and might to a certain extent be reflecting her mother's neurotic behaviour. She was trans- ferred to an open-air school, where some initial improvement was soon followed by a further pronounced deterioration in the standard of presentation of her work (Figs. 3 and 4). At the age of 8 both the parents and the schoolteacher thought that she was not seeing well.

At the school eye clinic her vision was found to be approximately $6 / 24$ ("E" test) in each eye. There was no significant error of refraction. An ophthalmic opinion was sought, but no organic basis could be found to explain the poor results of acuity testing and it was thought that this might be caused by her inability to co-

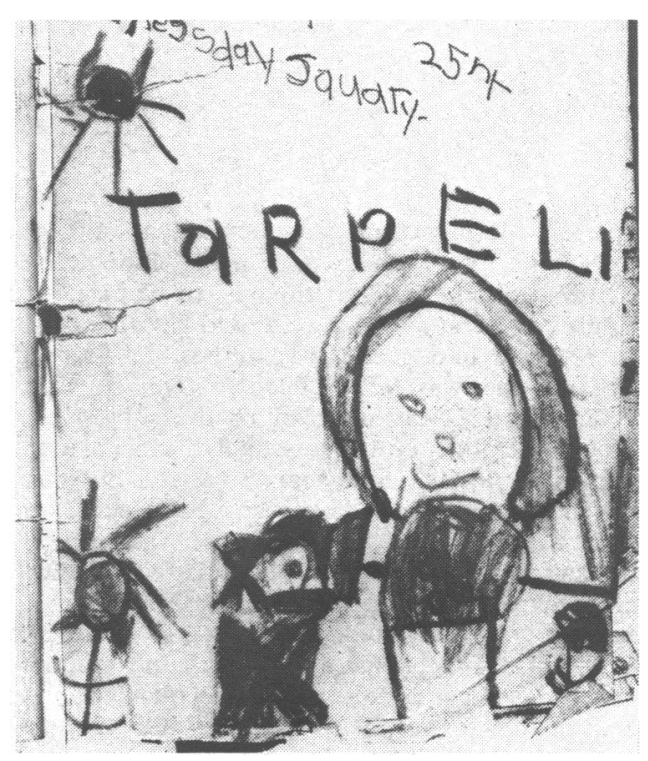

FIG. 3-Case 1. Specimen of school work at the age of 6 .

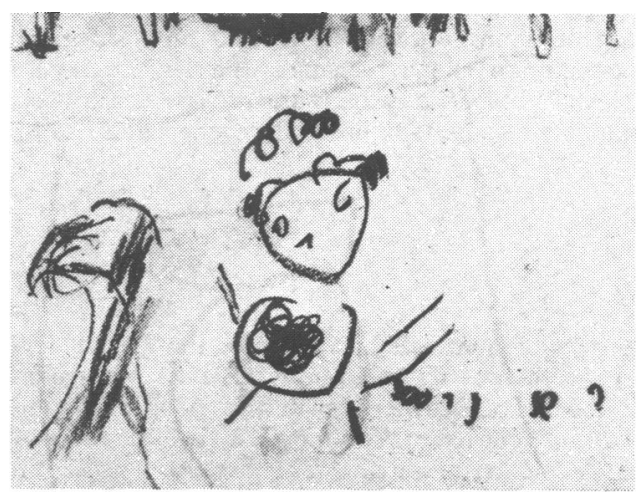

FIG. 4-Case 1. Specimen of school work at the age of 81

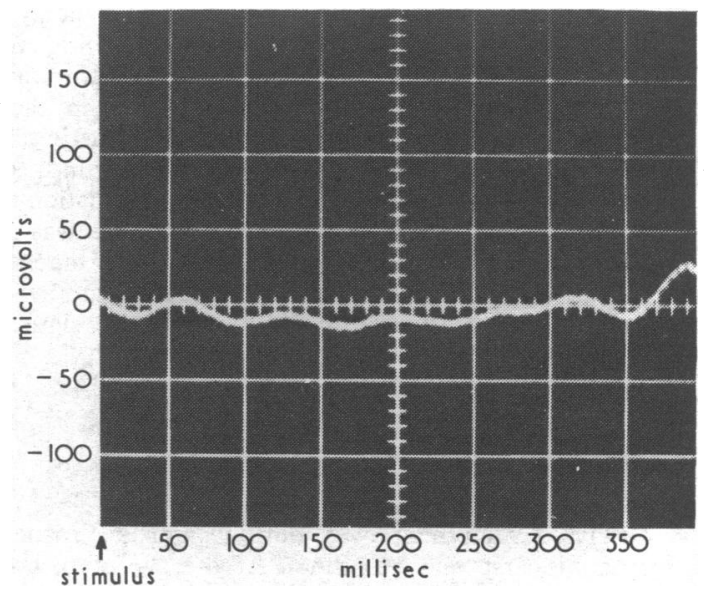

FIG. 5-Case 1. E.R.G. when fully dark-adapted at the age of 9. 
operate in the test. However, during the next six months her visual defect became much more obvious when she started sitting close to the television and falling over furniture. The visual acuity had now fallen to 6/60 ("E" test) in each eye, and fundus examination showed pallor of the optic discs, attenuation of the retinal arteries, and a fine granular pigmentary disturbance at the maculae. A diagnosis of tapetoretinal degeneration was made. The child was referred for a second ophthalmic opinion at the age of 9. At that time she was hardly able to see to eat her food. No fixation or following reflexes could be elicited. There was no nystagmus, but the pupillary reactions to light were sluggish. Fundus examination showed a more widespread pigmentary disturbance of the retina. The E.R.G. response was completely extinguished (Fig. 5), confirming the diagnosis of tapetoretinal degeneration.

\section{Case 2}

This boy's early development was normal, though the parents thought him a little slow compared with his siblings. At school he made very little progress and was thought to be backward. Routine school testing of sight and hearing showed no abnormality. At the age of 6 he broke a femur and was in hospital for seven weeks. During this time he was very uncooperative, spitting, throwing things, and crying. He settled down in the subsequent convalescence but soon after his return to school his behaviour again became disturbed, unpleasant, and aggressive, and he started to play truant. His school work deteriorated and his teacher thought that his vision might be defective, though he was not referred for ophthalmic examination, while at home it was noticed that he was becoming increasingly afraid of the dark.

When he finally refused altogether to go to school a psychiatric opinion was sought. It was considered that the emotional trauma associated with his fracture and the subsequent stay in hospital might be responsible for his behaviour. His full scale I.Q. was 63, the verbal scale 77 , and the performance scale 59. Ophthalmic examination at the age of $7 \frac{1}{2}$ showed that his visual acuity was only counting fingers in each eye, that the fields of vision were grossly constricted, and that he viewed things eccentrically, looking over the top of them. The pupils reacted only sluggishly to light. Fundus examination showed changes similar to those in Case 1 and the E.R.G. response was found to be totally extinguished, confirming a diagnosis of tapetoretinal degeneration.

\section{Case 3}

This boy's development was normal until the age of 6 years, when his school progress was found to be well below average and his concentration poor. The teachers suspected poor vision and he was referred for an ophthalmic opinion. A subjective assessment of visual acuity could not be made, but there was no significant error of refraction and no abnormality of the ocular media and fundi. Subsequently he became increasingly withdrawn and aggressive and was referred for a psychiatric opinion. His full scale I.Q. was 75, and no organic or environmental cause could be found for the behavioural disturbance, which was considered most likely to be hysterical in origin. By the age of 8 he was having obvious difficulty in avoiding obstacles, especially when the light was poor. Ophthalmic examination showed visual acuity of $6 / 9$ in each eye, gross constriction of the peripheral fields, and eccentric fixation. The pupillary reactions to light were sluggish. The fundi showed only slight pallor of the optic discs and some attenuation of the retinal arteries, but on electroretinography no response was elicited even with intense light stimuli. A diagnosis of tapetoretinal degeneration was made which was subsequently confirmed by full development of the characteristic ophthalmoscopic signs.

\section{Case 4}

This boy's early development was normal and he made good progress during his first year at school. At the age of 6, however, his work deteriorated and the teacher suspected poor vision. On ophthalmic examination visual acuity was found to be only $6 / 60$ in each eye, but there was no significant error of refraction and the ocular media and fundi were normal. As the child was becoming very withdrawn, bad tempered, and difficult to manage he was referred for a psychiatric opinion. The full scale I.Q. was 99 and no organic or environmental cause could be found for his behavioural disturbance which, it was thought, might be a hysterical manifestation. Because of increasing clumsiness and difficulty in avoiding objects, particularly if the light was poor, a second ophthalmic opinion was sought at the age of $7 \frac{1}{2}$. By now the visual acuity was only counting fingers and N.36 in each eye, with severe constriction of the peripheral fields and eccentric fixation. The appearances of the fundi were similar to those in Case 1 and no E.R.G. response could be elicited, confirming a diagnosis of tapetoretinal degeneration.

\section{Discussion}

The most striking feature of the history of each of these patients is the long interval, ranging from 18 to 30 months, between the onset of the disturbance of behaviour at the age of 6 years and the diagnosis of tapetoretinal degeneration. Cases 1,3 , and 4 had been suspected of having defective vision and had been referred for an ophthalmic opinion at a relatively early stage. No ocular abnormality could be found on routine clinical examination and their poor performance in visual acuity tests was attributed to their inability to co-operate. It was not until they were having obvious difficulty in getting around that further ophthalmic examinations led to the correct diagnosis. Case 2 had always been slightly retarded and his behavioural disturbance was very pronounced and overshadowed the visual defect. As a result an ophthalmic opinion was not sought until the age of $7 \frac{1}{2}$, by which time there was ophthalmoscopic evidence of tapetoretinal degeneration.

Two patients (Cases 3 and 4) were noticed to have more difficulty in walking around if the light was poor and the other two (Cases 1 and 2) were much more disturbed in the dark. All but Case 1 showed a characteristic form of eccentric fixation at the time of diagnosis. They seemed to look over the top of objects of regard, fixing with a suprafoveal area of the retina. At the time of the final diagnosis each patient had ophthalmoscopic signs of tapetoretinal degeneration which were confined to pallor of the optic discs, attenuation of the retinal arteries, and (except in Case 3) pigmentary disturbances at the macular areas. The E.R.G. was extinguished in all patients, confirming a diagnosis which at this late stage could be made on clinical grounds alone. However, an early and pronounced reduction or extinction of the E.R.G. is a constant feature of all forms of widespread tapetoretinal degeneration (Burian, 1962; Goodman et al., 1963) and the diagnosis can be made with confidence on this basis in the early stages of the congenital and juvenile forms of the disorder when fundus examination shows no abnormality.

Each child had been seen by a psychiatrist before the diagnosis of tapetoretinal degeneration was made. A diagnosis of hysteria was made in Cases 3 and 4 and Case 1 was thought to be semiautistic. Case 2 had obvious poor sight at the time of his psychiatric referral, but it was thought that his disturbance could be partially attributed to a fracture of his femur and the subsequent stay in hospital. All the patients have shown a considerable improvement in their patterns of behaviour since they settled down in schools for the visually handicapped and were no longer being forced to cope with the intolerable situation of competing with normally sighted children.

\section{Conclusion}

If a child is developing behavioural problems which could possibly be attributed to defective vision, then despite normal 
ocular appearances it is essential that electroretinography should be carried out as soon as possible to make or refute the diagnosis of tapetoretinal degeneration. This is the only way of eliminating the considerable period which may elapse before the diagnosis becomes evident on clinical examination, during which time an increasingly severe disturbance of behaviour may occur.

\section{References}

Burian, H. M. (1962). International Ophthalmology Clinics, 2, 21.

Goodman, G., Ripps, H., and Siegel, I. (1963). International Ophthalmology Clinics, 3, 777.

Harcourt, B. (1970). Developmental Medicine and Child Neurology, 12, 775. Karpe, G. (1945). Acta Ophthalmologica, Suppl. No. 24.

Leber, T. (1916). Graefe-Saemische-Hess Handbuch der gesamten Augenheilkunde, 2nd edn, vol. 7A, 2, p. 1076. Leipzig, Engelmann.

Noell, W. K. (1959). American fournal of Ophthalmology, 48, 347.

\title{
Hyperlactataemia in Phenformin-treated Diabetics
}

\author{
S. K. VARMA， S. J. HEANEY， W. G. WHYTE， R. S. WALKER
}

British Medical fournal, 1972, 1, 205-206

\section{Summary}

Raised fasting blood lactate levels were observed in diabetic patients on phenformin in therapeutic dosage. After an intravenous glucose load this effect was exaggerated and the lactate/pyruvate ratio increased. Withdrawal of the drug led to normal blood lactate levels and $a$ fall in the lactate/pyruvate ratio.

\section{Introduction}

Our interest in this field was stimulated by the admission of an elderly diabetic patient who had been treated with phenformin in a dose of $150 \mathrm{mg}$ daily. She was found to have hyperlactataemia and was acidotic. After withdrawal of phenformin, when the patient had recovered and was on dietary treatment alone, an oral glucose tolerance test with $50 \mathrm{~g}$ of glucose was carried out. Phenformin was then reintroduced and the dose built up to $150 \mathrm{mg} /$ day. The glucose tolerance test was repeated. Levels of glucose and blood lactate are shown in Table I. Blood sugar levels were satisfactory but lactate levels became high. This seemed to show a relation between the use of phenformin at this dosage level and a fall in blood sugar with a coincident hyperlactataemia.

Law Hospital, Carluke, Lanarkshire

S. K. VARMA, M.B., B.S., Medical Registrar

S. J. HEANEY, M.B., CH.B., Medical Registrar

W. G. WHYTE, M.B., CH.B., F.R.C.P., Consultant Physician

R. S. WALKER, M.D., F.R.C.P., Consultant Physician
It was therefore decided to investigate the problems further in other patients by using glucose intravenously to avoid any variation in absorption and to produce a high glucose load.

\section{Patients and Methods}

Twelve patients attending the diabetic clinic were chosen. Each had had a diabetic glucose tolerance test at the start of treatment. They were reasonably stabilized on phenformin $150 \mathrm{mg} /$ day and a daily diet containing $150 \mathrm{~g}$ of carbohydrate. Treatment with phenformin and the duration of diabetes varied from a few months to several years (Table II). Routine blood sugar levels one-and-a-half to two hours after a meal on their last three attendances at the clinic were less than $180 \mathrm{mg} / 100 \mathrm{ml}$. There was no evidence of significant renal or liver disease and they were having no other drugs. The patients were admitted to hospital and following an overnight fast venous blood samples were collected (with minimal constriction) before, and $5,15,30,60$, and 120 minutes after giving glucose $(25 \mathrm{~g}$ in $50 \%$ solution, intravenously. In Cases 9 to 12 a further intravenous glucose tolerance test was carried out after phenformin had been withdrawn for at least a week and while the patients were on a diet containing $150 \mathrm{~g}$ of carbohydrate.

In each sample true glucose was estimated, and lactate and pyruvate were measured by the Sigma (1965) method, the blood samples having been deproteinized immediately.

\section{Results}

The results are given in Tables III and IV. In the 12 patients on phenformin the average fasting lactate level was $15 \cdot 4$ (range

TABLE I-Oral Glucose Tolerance Test in Elderly Diabetic

\begin{tabular}{|c|c|c|c|c|c|c|c|c|c|c|c|c|}
\hline & \multicolumn{6}{|c|}{ Glucose $\mathrm{mg} / 100 \mathrm{ml}$} & \multicolumn{6}{|c|}{ Lactate $\mathrm{mg} / 100 \mathrm{ml}$} \\
\hline & \multirow{2}{*}{ Fasting } & \multicolumn{5}{|c|}{ Minutes } & \multirow{2}{*}{ Fasting } & \multicolumn{5}{|c|}{ Minutes } \\
\hline & & 30 & 60 & 90 & 120 & 150 & & 30 & 60 & 90 & 120 & 150 \\
\hline
\end{tabular}

table II-Details of Patients in Series

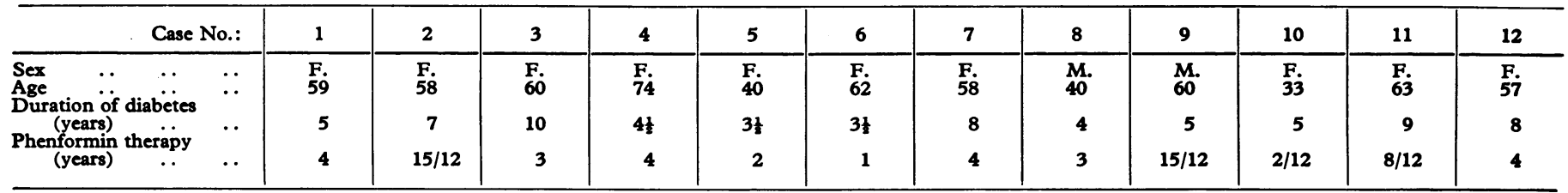

